\title{
Depression recognized as a risk factor in ACS
}

A scientific statement from the AHA recommends that depression be recognized as a risk factor for poor prognosis in patients with an acute coronary syndrome (ACS). The committee of experts performed a systematic review of the published literature and concluded that the preponderance of evidence indicated that depression is associated with adverse medical outcomes after ACS.

Approximately $20 \%$ of patients hospitalized because of an ACS (including myocardial infarction and unstable angina) meet the formal criteria for major depression, and many more have subclinical levels of depressive symptoms. By contrast, about $4 \%$ of the general US population is thought to have major depression. The committee's aim was to determine whether the association between depression and increased morbidity and mortality after ACS was sufficiently robust to identify depression as a risk factor for poor prognosis.

In a comprehensive literature search, 53 studies that met the inclusion criteria were identified. Of these studies, 32 reported on associations between depression and allcause mortality, 12 measured the association with cardiac mortality, and 22 contained a composite outcome that included mortality and nonfatal events. Depression was morecommonly measured by self-reporting than by interviews. The Beck Depression Inventory-I was most-frequently used.

The 32 studies in which all-cause mortality was used as an outcome measure involved 22 unique cohorts of

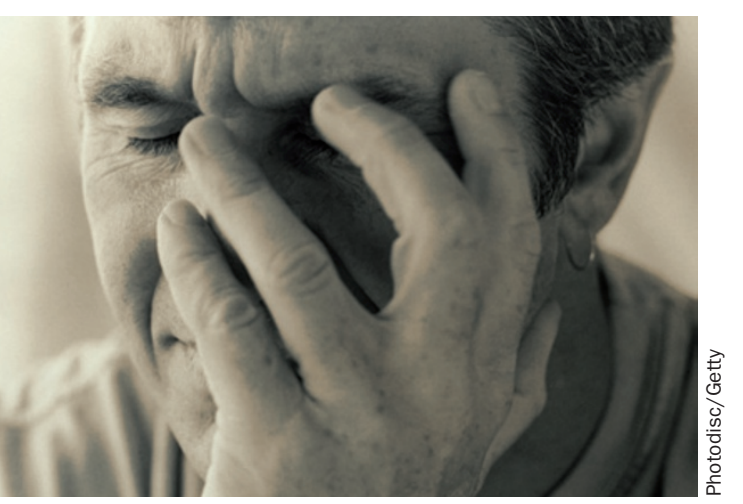

patients with ACS. Of the 32 studies, 17 showed a significant, risk-adjusted association between at least one measure of depression and increased all-cause mortality. A further four studies showed a significant unadjusted association.

A total of eight unique cohorts were involved in the 12 studies that measured cardiac mortality. In seven of these 12 studies, the risk-adjusted association between at least one measure of depression and increased cardiac mortality was significant. In one other study, a significant unadjusted association was reported.

In the 22 studies that included a composite outcome of mortality and nonfatal events, 18 unique cohorts were examined. The composite end point was either cardiac mortality and rehospitalization for a cardiac diagnosis (18 studies) or all-cause mortality and cardiac rehospitalization (four studies). In 15 of the 22 studies, a significant riskadjusted association between at least one measure of depression and increased mortality or nonfatal cardiac events was reported. Two other studies showed a significant unadjusted association.

The authors of the statement also identified four meta-analyses of the relationship between depression and mortality in patients with coronary heart disease. Three incorporated studies published up to the end of 2003; the other meta-analysis included studies up to the end of January 2011. In the three early meta-analyses, the overall, unadjusted effect of depression was 1.8-2.6 for all-cause mortality, and 2.3-2.9 for cardiac mortality. In the latest meta-analysis (limited to patients with myocardial infarction), the unadjusted effect of depression was 2.3 for all-cause mortality, 2.7 for cardiac mortality, and 1.6 for a composite outcome of fatal and nonfatal events.

On the basis of the evidence, the authors of the statement concluded that "depression is associated with increased ... mortality in patients with myocardial infarction". However, their review was hampered by the heterogeneity of the studies identified. The inclusion criteria, methods of assessing depression, subcategorization of different types of depression, and adequacy of risk adjustment varied between studies.

The formal identification of a risk factor requires the existence of a plausible biological mechanism. Although beyond the scope of their review, the authors suggest that depression might be associated with neuroendocrine dysfunction and disturbances in autonomic cardiac control, enhanced platelet activity, endothelial dysfunction, inflammation, or a combination of these mechanisms. Depression might also be associated with high-risk behaviour, such as smoking, a sedentary lifestyle, delay in seeking treatment, and nonadherence to secondary prevention measures. Notably, the authors did not require evidence that reducing the risk factor (that is, treating depression) improved prognosis after ACS. Data on this effect are scarce, and randomized clinical trials are warranted.

Andrew Steptoe, British Heart Foundation Professor from University College London, UK, who was not involved in writing the statement, points out that "the authors explicitly do not discuss the implications of this research for the screening of cardiac patients for depression, or for the management of depression. Both of these areas are controversial, with concern about whether screening is costeffective, and with little evidence to date that treating depression improves cardiac outcomes." Nonetheless, Professor Steptoe agrees that the statement provides "strong evidence that depression is related to worse outcomes even when clinical cardiological factors are taken into account".

Gregory B. Lim

Original article Lichtman, J. H. et al. Depression as a risk factor for poor prognosis among patients with acute coronary syndrome: systematic review and recommendations: a scientific statement from the American Heart Association. Circulation doi:10.1161/ CIR.0000000000000019 